\title{
On the Diffusion of an Ion Sheet in Poiseuille Flow
}

\author{
Stephen Jarvis, Jr.* \\ Institute for Basic Standards, National Bureau of Standards, Boulder, Colo. 80302
}

(March 1, 1968)

\begin{abstract}
A nonuniform sheet of ions generated at time $t=0$ diffuses in a cylindrical ion diffusion tube containing a nonreacting neutral species flowing with parabolic velocity distribution. Calculation of the on-axis ion density at a point $z$ downstream as a function of time $t$ is reduced to a single numerical integration for each $(z, t)$ involving some functions which have been computed once for all. An example is given showing the effect of the velocity distribution compared with a uniform flow with the same flow rate. The results appear to be corroborated by experiment.
\end{abstract}

Key Words: Diffusion, ion flow tubes.

\section{Introduction}

The analysis presented here is currently being used to predict the behavior of a diffusing ion sheet in a flow tube. In the experiment [5], ${ }^{1}$ an inert buffer gas, usually helium, is injected into a long flow tube about $8 \mathrm{~cm}$ in diameter. Distances along the tube are measured from a point $z=0$ downstream at which the flow has become essentially laminar, with a mean velocity of $8(10)^{3} \mathrm{~cm} / \mathrm{s}$ and a pressure of 0.4 torr. A thin sheet of ions is generated at $z=0$ at time $t=0$ by a pulsed beam of electrons incident perpendicular to the axis of the tube. The pulse width is variable, but $100 \mu \mathrm{s}$ might be considered characteristic. The relatively high pressure in the tube insures that the ions created will be thermalized at gas temperature in a few centimeters. The ion sheet is carried downstream by the flowing gas with a pulse shape determined by diffusion and the nonuniform velocity profile.

The sampling port of a mass spectrometer is located on the axis at a variable distance $z$ downstream from the electron gun, where $z$ is of the order of $100 \mathrm{~cm}$. The detector is gated with a gate width of $100 \mu \mathrm{s}$. Thus the ion arrival spectrum as a function of delay time between the termination of the excitation pulse and the initiation of the detection pulse is measured. Using experimentally measured values for the diffusion coefficient $D$, the computed arrival spectrum agrees well with the actual measured spectrum, indicating no serious errors are present in the formulation and solution of the transport equation.

Letting $(r, \theta)$ be coordinates in the cross section of the tube, the problem is to determine the time-dependent ion density $G(r, \theta, z, t)$ on the axis $(r=0)$ at a given distance $z$ downstream from the point at which the ions were generated as a thin sheet with given $(r, \theta)$-dependence. The background flow is assumed to be laminar and steady, with a parabolic velocity distribution, containing perhaps a neutral reactant species of uniform density $F$ everywhere. With a reaction rate $\kappa, G$ obeys the differential equation:

$$
\frac{\partial G}{\partial t}+v(r) \frac{\partial G}{\partial z}=D \mathscr{L} G-\kappa F G,
$$

${ }^{*}$ Consultant in mathematics, Radio Standards Laboratory, National Bureau of Standards, Boulder, Colo. 80302.

' Figures in brackets indicate the literature references on page 
where $\mathscr{L}$ is the Laplacian in cylindrical coordinates. Here $v(r)$ is the parabolic velocity distribution:

$$
v(r)=2 V\left(1-\left(\frac{r}{a}\right)^{2}\right),
$$

where $V$ is the mean velocity and $a$ is the tube radius. At the wall of the tube, the ion density is assumed to be negligible:

$$
G(a, \theta, z, t)=0 .
$$

We shall take for the given initial ion density distribution:

$$
G(r, \theta, z, 0)=P(r, \theta) \delta(z) .
$$

We notice that if $\kappa F=0$, the resulting solution $G_{0}(r, \theta, z, t)$ gives the general solution $G$ for $\kappa F \neq 0$ as follows:

$$
G(r, \theta, z, t)=G_{0}(r, \theta, z, t) e^{-\kappa F t},
$$

so that we may take $\kappa F=0$ in (1) without loss of generality.

\section{Solution by Integral Transform}

We shall expand $G$ by Laplace transform in $t$, Fourier transform in $z$, and an azimuthal expansion in $\theta$.

$$
\left\{\begin{array}{l}
G(r, \theta, z, t)=\frac{1}{(2 \pi)^{2}} \int_{-\infty}^{\infty} d k e^{i k z} \frac{1}{2 \pi i} \int_{c-i \infty}^{c+i \infty} d s e^{s t} \sum_{n=-\infty}^{\infty} e^{i n \theta} F_{n}(r ; k, s), \\
F_{n}(r ; k, s)=\int_{-\infty}^{\infty} d z e^{-i k z} \int_{0}^{\infty} d t e^{-s t} \int_{0}^{2 \pi} d \theta e^{-i n \theta} G(r, \theta, z, t) .
\end{array}\right.
$$

Then

$$
\frac{\partial^{2} F_{n}}{\partial r^{2}}+\frac{1}{r} \frac{\partial F_{n}}{\partial r}-\left[\frac{n^{2}}{r}+k^{2}+\frac{s+i k v(r)}{D}\right] F_{n}=-\frac{1}{D} P_{n}(\boldsymbol{r})
$$

where

$$
P_{n}(r)=\int_{0}^{2 \pi} d \theta P(r, \theta)
$$

We introduce the variables

$$
\left\{\begin{array}{l}
F_{n}(r)=\eta^{n} e^{-\eta / 2} H_{n}(\eta) \\
P_{n}(r)=q_{n}(\eta) \\
\eta=\hat{\gamma} r^{2}
\end{array}\right.
$$

where

$$
\hat{\gamma}^{2}=\frac{8 V}{a^{2} D}(-i k) .
$$


We obtain the inhomogeneous confluent hypergeometric equation [la]

$$
\eta H_{n}^{\prime \prime}+(1+2 n-\eta) H_{n}^{\prime}-\hat{a}_{n} H_{n}=-\frac{1}{-4 \hat{\gamma} D} \cdot \frac{q_{n}(\eta) e^{\eta / 2}}{\eta^{n}}
$$

where

$$
\hat{a}_{n}=n+\frac{1}{2}+\frac{1}{4 \hat{\gamma}}\left[k^{2}+\frac{s+2 i k V}{D}\right]
$$

The homogeneous equation has the solutions $[1 \mathrm{~b}, 1 \mathrm{c}]$

$$
\phi\left(\hat{a}_{n}, 1+2 n ; \eta\right), \psi\left(\hat{a}_{n}, 1+2 n ; \eta\right),
$$

with Wronskian

$$
W=-\frac{e^{\eta} \Gamma(1+2 n)}{\eta^{1+2 n} \Gamma\left(\hat{a}_{n}\right)}
$$

As must be expected, only the lowest azimuthal modes, $n=0$, contribute to the axial value $r=0$ of the ion density. The solution satisfying smoothness at $\eta=0$ and vanishing at $\eta=\hat{\gamma} a^{2}$ is, for $n=0$ :

$$
F_{0}(r)=\frac{\Gamma\left(\hat{a}_{0}\right)}{4 \hat{\gamma} D} e^{-\eta / 2} \cdot\left\{\frac{\mathscr{G}\left(\hat{\gamma} a^{2}\right)}{\phi\left(\hat{a}_{0}, 1 ; \hat{\gamma} a^{2}\right)} \phi\left(\hat{a}_{0}, 1 ; \eta\right)-\mathscr{G}(\eta)\right\}
$$

where

$$
\mathscr{G}(\eta)=\phi\left(\hat{a}_{0}, 1 ; \eta\right) \int_{0}^{\eta} d t t e^{-\frac{1}{2} t} q_{0}(t) \psi\left(\hat{a}_{0}, 1 ; t\right)-\psi\left(\hat{a}_{0}, 1 ; \eta\right) \int_{0}^{\eta} d t t e^{-\frac{1}{2} t} q_{0}(t) \phi\left(\hat{a}_{0}, 1 ; t\right)
$$

On $r=0$,

$$
F_{0}(0)=\frac{1}{4 \hat{\gamma} D} \Gamma\left(\hat{a}_{0}\right) \mathscr{G}\left(\hat{\gamma} a^{2}\right) / \phi\left(\hat{a}_{0}, 1 ; \hat{\gamma} a^{2}\right)
$$

If we write

$$
b=a^{2} \hat{\gamma}
$$

and

$$
\mathscr{L}_{(s, t)}^{-1} F_{0}(0 ; k, s) \equiv \chi(t ; k)
$$

for the inverse Laplace transform, (17) gives

$\chi(t ; k)=\frac{a^{2}}{4 D} \int_{0}^{1} d z e^{-\frac{b z}{2}} q_{0}(b z) \mathscr{L}_{(s, t)}\left\{\Gamma\left(\hat{a}_{0}\right) \cdot \psi\left(\hat{a}_{0}, 1 ; b z\right)-\Gamma\left(\hat{a}_{0}\right) \frac{\psi\left(\hat{a}_{0}, 1 ; b\right) \phi\left(\hat{a}_{0}, 1 ; b z\right)}{\phi\left(\hat{a}_{0}, 1 ; b\right)}\right\}$.

We put

$$
\begin{gathered}
A=\frac{a^{2}}{4 b D} s, \quad \tau=\frac{4 D}{a^{2}} t, \\
\hat{\nu}=\frac{a^{2}}{4} k^{2}+\frac{a^{2} V}{2 D} i k,
\end{gathered}
$$


and (19) becomes by translation

$$
\chi(t ; k)=b e^{-\left(\hat{v}+\frac{b}{2}\right) \tau} \int_{0}^{1} d z e^{-\frac{b}{2} z} q_{0}(b z) \mathscr{L}_{(A, b \tau)}^{-1} \cdot\left\{\Gamma(A) \psi(A, 1 ; b z)-\Gamma(A) \psi(A, 1 ; b) \frac{\phi(A, 1 ; b z)}{\phi(A, 1 ; b)}\right\}
$$

The $\mathscr{L}^{-1}$ of the first term in the bracket can be found in tables [2a], when $\phi$ is replaced by the proper Wittaker function, or may be found by the residue theorem from the poles of $\Gamma(A)$ at $A=0,-1,-2, \ldots$. . The second term may be evaluated in two parts. First, the poles of $\Gamma(A)$ are shown in the appendix 1 to give a term which exactly cancels the contribution from the first term in the bracket. Second, the zeroes of $\phi(A, 1 ; b)$, which we shall designate as $a_{m}(b) ;(m=1,2, \ldots)$,

$$
\phi\left(a_{m}(b), 1 ; b\right)=0
$$

give by the residue theorem

$$
\begin{aligned}
\chi(t ; k)=e^{-\hat{\nu} \tau} \Gamma(b, \tau), \Gamma(b, \tau)=\sum_{m=1}^{\infty} \Gamma_{m}(b, \tau), & \\
& \Gamma_{m}(b, \tau) \equiv-b e^{-\frac{b \tau}{2}} \frac{\Gamma\left(a_{m}\right) \psi\left(a_{m}, 1 ; b\right)}{\phi, \hat{a}_{0}\left(a_{m}, 1 ; b\right)} e^{a_{m} b \tau} \cdot \sum_{K=0}^{\infty} \frac{\left(a_{m}\right)_{K} b^{K}}{(K !)^{2}} \mathscr{I}_{K} .
\end{aligned}
$$

The last factor in $\Gamma_{m}(b, \tau)$ comes from integration with respect to $z$ using the power series for $\phi\left(a_{m}, 1 ; b z\right)$ :

$$
\mathscr{I}_{K}\left(b ; P_{0}\right) \equiv \int_{0}^{1} d z e^{-\frac{b}{2} z} z^{K} P_{0}(a \sqrt{z})
$$

It is important to note that when we let $b \rightarrow 0$ in the above expression, we find

$$
\sum_{K=0}^{\infty} \frac{\left(a_{m}\right)_{K} b^{K}}{(K !)^{2}} \mathscr{I}_{K}(b) \rightarrow 2 \int_{0}^{1} d z z J_{0}\left(\xi_{m} z\right) P_{0}(a z),
$$

using the limiting forms for $\phi$ in terms of $I_{0}$ as $b \rightarrow 0, a_{m} b$ finite: [1d]

$$
\begin{gathered}
\phi\left(\hat{a}, 1 ; \frac{(b \hat{a})}{\hat{a}}\right) \underset{\hat{a} \rightarrow \infty}{ } I_{0}(2 \sqrt{b \hat{a}}) . \\
|b \hat{a}|<\infty
\end{gathered}
$$

This also gives the location of zeroes for small $b$ :

$$
a_{m}(b) \rightarrow-\xi_{m}^{2} / 4 b
$$

where $J_{0}\left(\xi_{m}\right)=0, \quad(m=1,2,3, \ldots)$. Indeed, as $b \rightarrow 0$, the entire expression (23) becomes independent of $b$, and the inverse Fourier transform involves only the factor $e^{-i \tau}$, from which we recover the solution corresponding to a uniform velocity $v(r) \equiv 2 V$. For $P_{0}(r) \equiv 2 \pi$, this is

$$
\left\{\begin{array}{l}
G(0, z, t)=\frac{1}{\sqrt{\pi D t}} e^{-\frac{(z-2 V t)^{2}}{4 D t}} \sum_{m=1}^{\infty} \frac{e^{-\xi_{m}^{2} D t / a^{2}}}{\xi_{m} J_{1}\left(\xi_{m}\right)}, \\
v(r) \equiv 2 V .
\end{array}\right.
$$


For $z>0$, the $(-i k)^{1 / 2}$ factor in $b$ is to be interpreted as $\lim _{l \rightarrow 0^{+}}(-l-i k)^{1 / 2},(l>0)$, so that the path of integration in the $k$-plane for the inverse Fourier transform passes below the branch at $k=0$ :

$$
-\pi \leqslant \arg k \leqslant 0
$$

We replace $k$ by $b$ through:

$$
k=\frac{D}{2 a^{2} V} b^{2} e^{i \frac{\pi}{2}}
$$

and take the path of integration $C$ in the $b$-plane to run from $\infty e^{-i \frac{3 \pi}{4}}$ to the origin, $C_{1}$, and out to $\infty e^{-i \frac{\pi}{4}}, C_{2}$.

Then with

$$
\gamma \equiv \frac{a V}{2 D}, \quad z \equiv \frac{D}{2 a^{2} V} z
$$

we have

$$
G(0, z, t)=\frac{1}{4 \pi a \gamma} \int_{C} d b i b \Gamma(b, \tau) e^{-b^{2}\left({ }^{z}-\frac{\tau}{4}\right)+\frac{1}{(8 \gamma)^{2}} b^{4} \tau} .
$$

Putting $b=u e^{-i \frac{3 \pi}{4}}$ on $C_{1}, b=u e^{-i \frac{\pi}{4}}$ on $C_{2}$, and noting that $G$ is real, we obtain

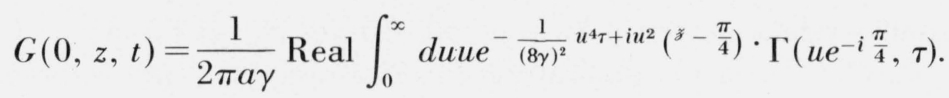

In a particular case of interest, $(8 \gamma)^{2}=3.7(10)^{3}, z \approx 0.3,\left|z-\frac{\tau}{4}\right| \leqslant 0.1$. For such values expansion methods are not satisfactory, especially in view of the difficulty of getting any simple expressions for the zero-curve $a_{m}(b)$ and $\Gamma(b, \tau)$. Numerical integration then seems to be necessary.

\section{Numerical Integration}

It is convenient to write

$$
G(0, z, t)=\sum_{m=1}^{\infty} G_{m}(z, t)
$$

where

$$
G_{m}(z, t)=\frac{1}{2 \pi a \gamma} \operatorname{Real} \int_{0}^{\infty} d u u e^{-\frac{1}{(8 \gamma)^{2}} u^{4} \tau+i u\left(\tilde{z}-\frac{\tau}{4}\right)} \cdot \Gamma_{m}\left(u e^{-i \frac{\pi}{4}}, \tau\right),
$$

where the subscript $m$ refers to the terms due the zeroes of $\phi\left(a_{m}(b), 1 ; u e^{-i \frac{\pi}{4}}\right)$ which, as $u \rightarrow 0$ are given in (26). For computational purposes, it is convenient to introduce the following functions:

$$
\left\{\begin{array}{l}
F(u, B) \equiv \phi\left(B / u, 1 ; u e^{-i \frac{\pi}{4}}\right), \\
u F,_{B}(u, B)=\phi, \hat{a}_{0}\left(B / u, 1 ; u e^{-i \frac{\pi}{4}}\right) .
\end{array}\right.
$$


Treating $u$ as a parameter, the complex zero curve for each $m$,

$$
\left\{\begin{array}{l}
F\left(u, B_{m}(u)\right)=0, \\
a_{m}(b)=B_{m}(u) / u,
\end{array}\right.
$$

is found by Newton-Raphson iteration

$$
\left\{\begin{array}{l}
B_{m}^{(n+1)}=B_{m}^{(n)}-\frac{F\left(u, B_{m}^{(n)}\right)}{F,{ }_{B}\left(u, B_{m}^{(n)}\right)} \\
B_{m}^{(0)}(0)=-\frac{1}{4} \xi_{m}^{2} e^{i \frac{\pi}{4}}
\end{array}\right.
$$

using the expansions [16]

$$
F(u, B) \doteq \sum_{K=0}^{K_{f}} Q_{K}(u, B),
$$

where

$$
\begin{gathered}
Q_{K}(u, B)=\frac{B(B+u) \ldots(B+(K-1) u)}{(K !)^{2}} e^{-i \frac{K \pi}{4}} \\
F,{ }_{B}(u, B) \doteq \sum_{K=0}^{K_{f}} Q_{K}(u, B) \cdot\left[\frac{1}{B}+\frac{1}{B+u}+\ldots+\frac{1}{B+(K-1) u}\right],
\end{gathered}
$$

with $K_{f}$ chosen to control relative error in $F_{B}$ at cutoff. Curves $B_{m}(u)$ for $m=1,2,3$ were computed for $0 \leqslant u \leqslant 17$ for a set of Gaussian quadrature abscissae on each unit interval in $u$.

For fixed $u$, the number $N$ of zeroes of $\phi\left(\frac{B(u)}{u}, 1 ; u e^{-i \frac{\pi}{4}}\right)$ inside a circle radius $\rho$ centered on $B_{0}$ is easily shown to be

$$
N=-\frac{\rho}{2} \int_{-1}^{1} d x e^{i \pi x} \frac{F,{ }_{B}\left(u, B_{0}-\rho e^{i \pi x}\right)}{F\left(u, B_{0}-\rho e^{i \pi x}\right)}
$$

Using Gaussian quadrature, it was easily established that no zeroes occur for $|B| \leqslant 10$, $0 \leqslant u \leqslant 17$, other than those continued from $B_{m}(0)$.

The remaining functions necessary to compute $\Gamma(b, \tau)$ are found from the following:

$$
\begin{gathered}
g_{m}(u) \equiv-\Gamma\left(a_{m}\right) \psi\left(a_{m}, 1 ; u e^{-i \frac{\pi}{4}}\right)=\sum_{K=0}^{K_{f}} Q_{K}\left(u, B_{m}\right)\left[\psi\left(\frac{B_{m}}{u}+K\right)-2 \psi(1+K)\right] \\
l_{m}\left(u ; P_{0}\right)=\sum_{K=0}^{K_{f}} Q_{K}\left(u, B_{m}\right) \mathscr{I}_{K}\left(u e^{-i \frac{\pi}{4}} ; P_{0}\right)
\end{gathered}
$$

where $\psi(z)$ is the Digamma function [3a] and $\mathscr{I}_{K}$ is defined in (27). $\psi(z)$ is computed by asymptotic series for $|z|>4$, and for $|z|<4$ the recursion relation is used to get $z$ outside this circle.

Clearly the radial distribution $P_{0}(r)$ occurs only in $l_{m}(u)$ via $\mathscr{I}_{K}$, and does not affect the zero-curve location. Then

$$
\Gamma_{m}\left(u e^{-i \frac{\pi}{4}}, \tau\right)=e^{-i \frac{\pi}{4}} e^{-\frac{b \tau}{2}} \frac{g_{m}(u) l_{m}\left(u ; P_{0}\right)}{F,{ }_{B}\left(u, B_{m}(u)\right)},
$$


and the numerical integration in (32) is carried out by Gaussian quadrature on the unit intervals of $u$.

From the discussion following (24), the distribution for uniform velocity profile $v(r) \equiv 2 \mathrm{~V}$ is

$$
[G(0, z, t)]_{\text {uniform }}=\frac{1}{2 \pi a \gamma} \text { Real } \cdot \int_{0}^{\infty} d u u e^{-\frac{1}{(8 \gamma)^{2}} u^{4} \tau+i u^{2}\left(z-\frac{\tau}{4}\right)} \Gamma(0 ; \tau) .
$$

\section{Results: $P_{0}(r) \equiv 2 \pi$}

Detailed calculations have been made only for the uniform radial distribution $P_{0}(r) \equiv 2 \pi$ for which (24) is readily evaluated by recursion. (In particular, the results apply to a uniform distribution $P(r, \theta)=1$ in (8)). A numerical evaluation for more general $P_{0}(r)$ is readily substituted. For typical numbers $(\tau, z, \gamma)$ of interest, the first term, $m=1$ in (33), dominated the second, $m=2$, by the factor $10^{4}$.

Figure 1 shows $B_{m}(u)$, the zero-curve, for $m=1,2$. Figure 2 shows $G(0, z, t)$ for $a=4 \mathrm{~cm}$, $D=1800 \mathrm{~cm}^{2} / \mathrm{s}, z=42 \mathrm{~cm}$, and a mean velocity $V=6800 \mathrm{~cm} / \mathrm{s}$ for both the parabolic and uniform velocity profiles.

Figure 3, taken from a forthcoming publication by Fehsenfeld [5], shows a comparison of the experimental profile (solid points) and the calculated profile (open points) for the values $a=4 \mathrm{~cm}$, $D=2400 \mathrm{~cm}^{2} / \mathrm{s}, z=113 \mathrm{~cm}$ and $V=6800 \mathrm{~cm} / \mathrm{s}$. In this figure, both sets of data are normalized to peak values of unity, and the time of the experimental peak is shifted by $0.3 \mathrm{~ms}$.

Computation of the zero-curve for each $m$ took three minutes, while the curves in figure 1 took two minutes each on the CDC 3600 with complex arithmetic hardware. A 50-point Gaussian integration was used throughout [4]. Fortran programs are available from the author.

The author wishes to express his thanks to Fred C. Fehsenheld and Arthur L. Schmeltekopf for suggesting the problem and providing experimental verification of the results.

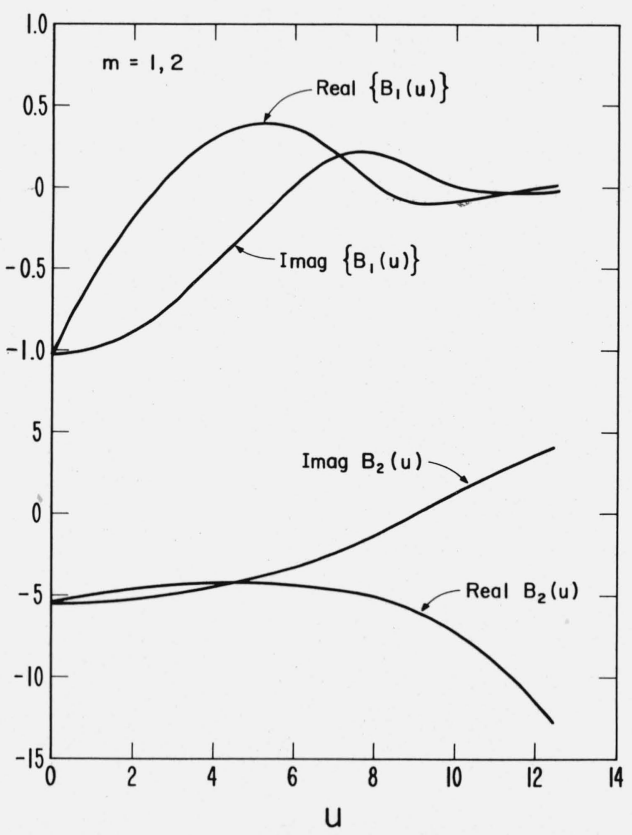

Figure 1. Zero curves $\mathrm{B}_{\mathrm{m}}(\mathrm{u})$ for $\mathrm{m}=1,2$ (eq 37$)$.

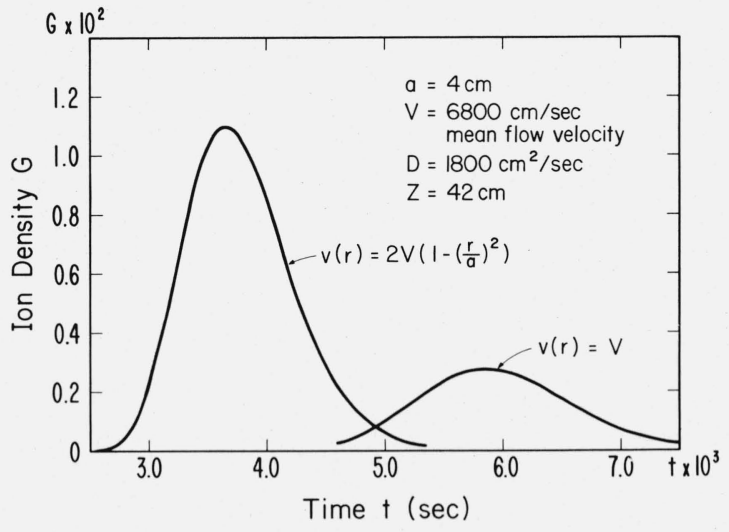

FIGURE 2. Comparison of sample ion densities for uniform and parabolic velocity profiles. 


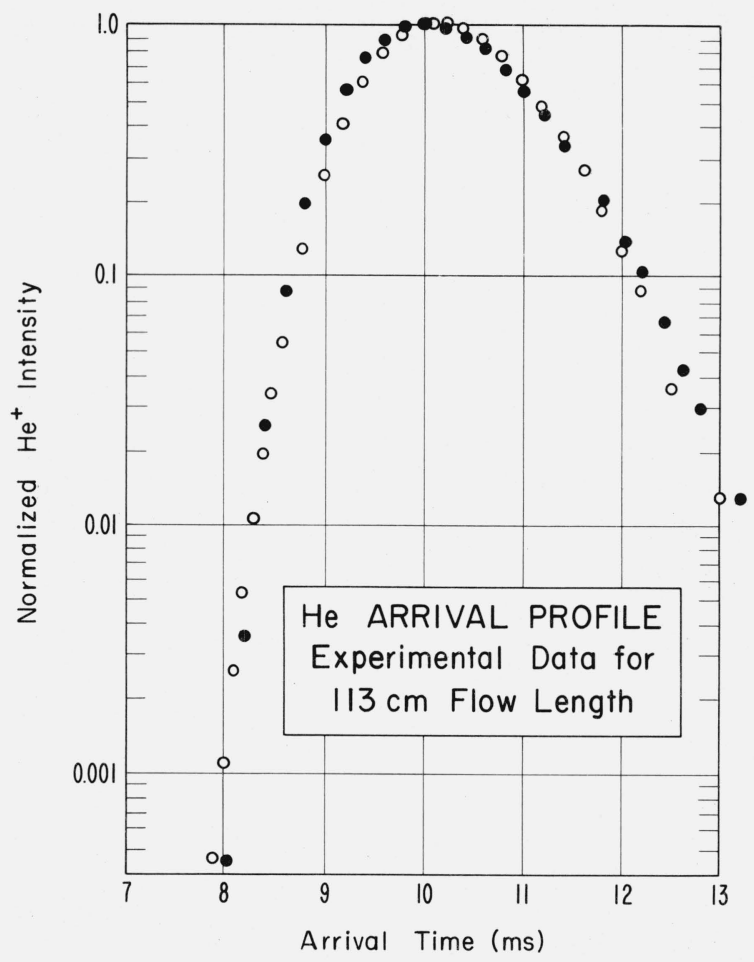

FIGURE 3. Comparison of normalized experimental and calculated profiles with juxtaposed peaks.

\section{References}

[1] Erdelyi, A., Higher Transcendental Functions, Bateman Manuscript Project 1; (a) 248 (2), (b) 248 (1), (c) 248 (7), (d) 266 (18), (e) 264 (2) (McGraw-Hill Book Co., Inc., New York, N.Y., 1954).

[2] Erdelyi, A., Tables of Integral Transforms, Bateman Manuscript Project 1; (a) 296 (23) [Note error pointed out in Appendix to this paper], (b) 147 (41) (McGraw-Hill Book Co., Inc., New York, N.Y., 1954).

[3] Abramowitz, M., and I. A. Stegun (1964), Handbook of Mathematical Functions, NBS AMS 55, (a) 259, (b) 784 (22.9.15) (1964).

[4] Love, C. H., Abscissas and weights for Gaussian quadrature for $N=2$ to 100 , and $N=125,150$, 175, and 200, J. Res. NBS 70B (Math. and Math. Phys.), No. 4, 249 (1966).

[5] Bates, D. R., and E. Estermann, Advances in Atomic and Molecular Physics, Vol. 5 (Academic Press, New York, London, to be published).

\section{Appendix}

We shall show that contributions to the inverse Laplace transform due to the poles of $\Gamma(A)$ in the two terms in (21) cancel out.

From well-known relations $[1 \mathrm{e}, 2 \mathrm{a}]$, the first term gives:

$\mathscr{L}_{(A, b \tau)}^{-1} \Gamma(A) \psi(A, 1 ; b z)=\mathscr{L}^{-1} e^{\frac{b z}{2}}(b z)^{-1 / 2} \Gamma(A) W_{1 / 2-A, 0}(b z)$

$$
=e^{b \tau}\left(e^{b \tau}-1\right)^{-1} \exp \left(\frac{-b z}{e^{b \tau}-1}\right)
$$

according to tables $[2 \mathrm{a}]$. (Note that in the reference [2a], second column, $\left(\frac{1}{2}-K-u\right) t$ should read $\left(\frac{1}{2}+K-u\right) t$ to agree with reference $[2 \mathrm{~b}]$ and the straightforward residue evaluation.) 
With regard to the second term, let us put

$$
\Phi(A) \equiv \frac{\phi(A, 1 ; b z)}{\phi(A, 1 ; b)}
$$

and assume that this factor does not contribute to $\mathscr{L}^{-1}$. (Its contributions are treated separately in the text.) Then for $c>1$ (the nonlogarithmic case) [lc]

$$
\begin{aligned}
\mathscr{L}_{(A, b \tau)}^{-1} \Gamma(A) \psi(A, 1 ; b) \Phi(A) \equiv Q(t ; b, c) & \\
=\frac{\pi}{\sin \pi c}\left\{\mathscr{L}^{-1} \frac{\Gamma(A) \phi(A, c ; b)}{\Gamma(c) \Gamma(1+A-c)} \Phi(A)\right. & \left.-b^{1-c} \mathscr{L}^{-1} \frac{\phi(1+A-c, 2-c ; b)}{\Gamma(2-c)} \Phi(A)\right\},
\end{aligned}
$$

by the definition of $\psi(A, c ; b)$ in terms of two independent solutions $\phi(A, c ; b)$ and $b^{1-c} \phi(1+A-c$; $2-c ; b)$ for noninteger $c$. The second term on the right of (A3) vanishes due to the regularity of the integrand, so that by the residue theorem, the poles of $\Gamma(A)$ give:

$$
Q(t ; b, c)=\frac{\pi}{\sin \pi c} \sum_{m=0}^{\infty}(-)^{m} \frac{\phi(-m, c ; b) e^{-m b \tau}}{m ! \Gamma(c) \Gamma(1-m-c)} \Phi(-m) .
$$

Taking the limit $c \rightarrow 1^{+}$,

$$
\begin{aligned}
& \phi(-m, c ; b) \rightarrow \phi(-m, 1 ; b), \\
& \Gamma(c) \rightarrow 1, \\
& \sin \pi c \Gamma(1-m-c) \rightarrow \frac{(-)^{m} \pi}{m !}, \quad\left(c \rightarrow 1^{+}\right) .
\end{aligned}
$$

We find then, that $c \rightarrow 1^{+}$,

$$
Q(t ; b, 1)=\sum_{m=0}^{\infty} \phi(-m, 1 ; b) e^{-m b \tau} \Phi(-m)
$$

Replacing $\Phi(-m)$ by (A2),

$$
Q(t ; b, 1)=\sum_{m=0}^{\infty} \phi(-m, 1 ; b z) e^{-m b \tau}
$$

By the formula for the generating function for the Laguerre polynomials [3b],

$$
\sum_{m=0}^{\infty} \phi(-m, 1 ; b) x^{m}=(1-x)^{-1} \exp \left(\frac{-b x}{1-x}\right)
$$

putting $x=e^{-b \tau}$, we obtain the equivalence of the expressions (Al) and (A6). 